\title{
Revisiting rhino-orbito-cerebral acute invasive fungal sinusitis in the era of COVID-19: pictorial review
}

\author{
Smita Manchanda ${ }^{1} \cdot$ Kapil Semalti $^{1} \cdot$ Ashu Seith Bhalla ${ }^{1}$ (D) Alok Thakar ${ }^{2} \cdot$ Kapil Sikka $^{2} \cdot$ Hitesh Verma $^{2}$
}

Received: 30 June 2021 / Accepted: 16 August 2021 / Published online: 31 August 2021

(c) American Society of Emergency Radiology 2021

\begin{abstract}
COVID-19 patients have been found to have an increased incidence of superadded fungal infections because of multiple factors such as impaired cell-mediated immunity, immunosuppressive therapy, and coexistent diabetes mellitus. Recently, there has been a significant rise in the COVID-19-associated mucormycosis and aspergillosis cases involving the sinonasal cavity and the lungs. Rhino-orbito-cerebral acute invasive fungal rhinosinusitis (AIFR) is a potentially life-threatening, invasive fungal infection. Early diagnosis followed by prompt medical management and surgical intervention is crucial for patient survival. The role of cross-sectional imaging (CT/MRI) is not only to suggest a diagnosis of invasive fungal sinusitis but also to delineate the complete extent of disease. Mapping the extent of orbital and intracranial disease has prognostic as well as management implications, as involvement of these sites marks a worse prognosis. A stepwise approach to evaluation of imaging of AIFR along with a pictorial depiction of the key imaging findings is presented.
\end{abstract}

Keywords COVID-19 $\cdot$ Mucormycosis $\cdot$ Invasive fungal infections $\cdot$ Sinusitis $\cdot$ Aspergillosis

\section{Introduction}

There has been a recent surge in the number of cases of COVID-19-associated mucormycosis and aspergillosis in the aftermath of the pandemic in several countries [1]. Rhino-orbito-cerebral mucormycosis (ROCM) is a form of acute, invasive fungal rhinosinusitis (AIFR) caused by

Ashu Seith Bhalla

ashubhalla1@yahoo.com

Smita Manchanda

smitamanchanda@gmail.com

Kapil Semalti

semaltikapil@gmail.com

Alok Thakar

drathakar@gmail.com

Kapil Sikka

kapil_sikka@yahoo.com

Hitesh Verma

drhitesh10@gmail.com

1 Department of Radiodiagnosis, All India Institute of Medical Sciences, New Delhi 110029, India

2 Department of Otorhinolaryngology \& Head-Neck Surgery, All India Institute of Medical Sciences, New Delhi 110029, India zygomycetes order Mucorales. The Mucor species can be a commensal in the nasal mucosa of healthy people and in the presence of immunosuppression, germinate within the nose and paranasal sinuses and further progress to ROCM. A decrease in $\mathrm{CD}^{+} \mathrm{T}$ and $\mathrm{CD} 8^{+} \mathrm{T}$ cells in COVID-19 patients leads to impaired cell-mediated immunity and an increased predisposition to fungal infections [2]. In addition, there is a complex interplay of factors including overexpression of inflammatory cytokines, immunosuppressive therapy, comorbidities like diabetes mellitus, and nosocomial infection sources leading to an increased incidence of AIFR. In the non-COVID setting, AIFR has been reported to be caused by many fungal species such as Mucor, Rhizopus, Rhizomucor, and Aspergillus [3]. However, in the context of COVID-19, most studies have reported Mucor species (COVID-19-associated mucormycosis/CAM). In a recent study by El-Kholy et al. [4], Mucor species was confirmed histopathologically in 28 patients, Aspergillus fumigatus in 11 , and coinfection in 3 cases.

COVID management teams should have a high index of clinical suspicion for AIFR, with all patients monitored for early warning signs and symptoms. These include nasal stuffiness; foul smell; epistaxis; black nasal discharge; nasal mucosal discolouration or eschar; locoregional pain or swelling; facial paresthesia; proptosis, ptosis, sudden loss of 
Fig. 1 Pre-existing chronic changes in different patients of COVID-19 with nasal stuffiness. Axial CT (a) reveals a welldefined soft tissue lesion (*) in the expanded left maxillary sinus (arrows) with extension into left nasal cavity through maxillary sinus ostium, suggestive of polyp. Coronal T2W MRI image of another patient (b) shows well-defined round hyperintense lesions (*) along the floor of bilateral maxillary sinuses suggestive of retention cysts. NCCT axial image (c) shows hypodense contents in left maxillary sinus with internal hyperdensity $(*)$ and coronal T2WI (d) shows hypointense contents (arrowhead) with surrounding hyperintense mucosal thickening (arrow) suggestive of Mycetoma
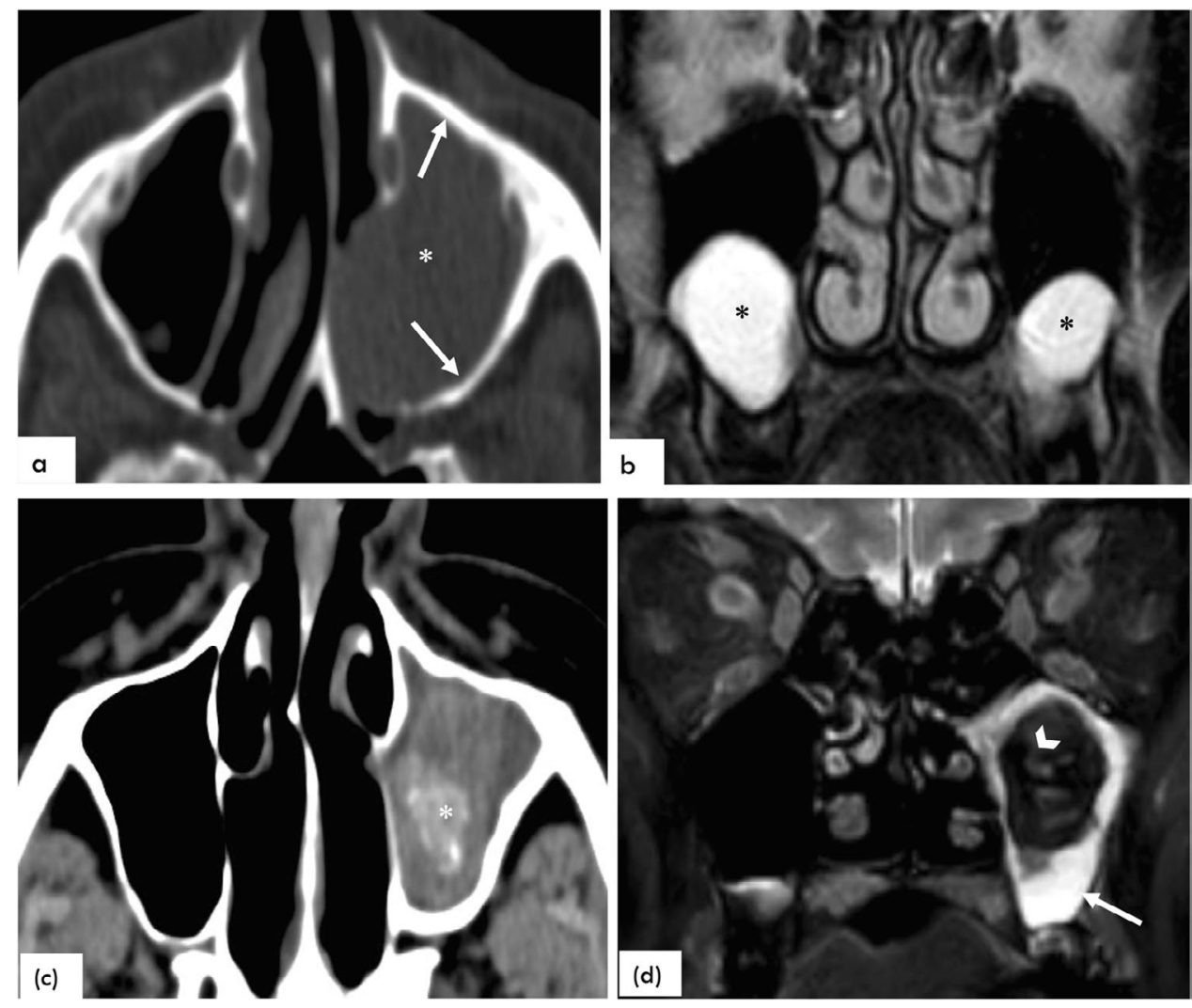

vision or diplopia; facial palsy; altered sensorium; paralysis; and focal seizures $[5,6]$. In the presence of this symptomatology, patients need to undergo clinico-radiological evaluation in the form of nasal endoscopy and contrast enhanced CT (CECT) or contrast enhanced MRI (CEMRI). We suggest a stepwise approach in the imaging evaluation of suspected rhino-orbito-cerebral AIFR with pictorial depiction of the key imaging findings.

\section{Discussion}

The diagnosis of AIFR is made clinically with nasal endoscopy and histopathological examination. The role of imaging in suspected AIFR is primarily for documenting the extent of disease and follow-up. CEMRI is the imaging modality of choice for suspected intracranial and intraorbital disease extension; however, it has the disadvantage of limited availability in several health set-ups, longer acquisition times, and

Table 1 Extent of sinonasal and perisinus disease in ROCM

\begin{tabular}{ll}
\hline Sinonasal disease & Perisinus disease \\
\hline Nasal cavity: & $\bullet$ Anterior periantral space \\
- Middle turbinate (erosion/soft tissue thickening/black turbinate sign) & $\bullet$ Posterior periantral space \\
- Inferior turbinate (erosion/soft tissue thickening/black turbinate sign) & $\bullet$ Buccal space: necrotic abscess \\
- Nasal septum (erosion) & $\bullet$ Zygoma and mandible: bony erosions/marrow edema \\
- Bilateral disease & $\bullet$ Soft tissue in nasopharynx, sphenopalatine foramen, pterygomaxillary \\
Paranasal sinuses: & fissure, pterygopalatine fossa, and infratemporal fossa \\
- Mucosal thickening/partial/complete opacification & \\
- Unilateral disease & \\
- One sinus & \\
- Multiple sinuses & \\
- Bilateral disease & \\
$\circ$ One sinus & \\
- Multiple sinuses & \\
- Erosion of palate & \\
Oronasal/oroantral fistula &
\end{tabular}




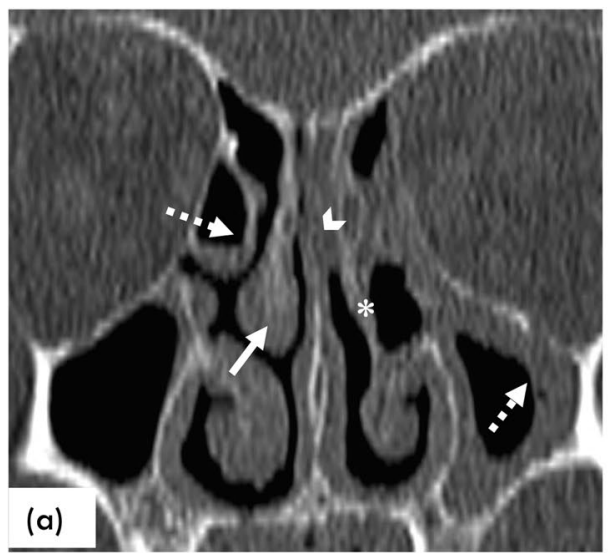

Fig. 2 CAM with nasal cavity and sinus involvement. CT coronal image in bone window (a) reveals soft tissue density in left nasal cavity (arrowhead) with erosion of left middle turbinate (*). Normal right middle turbinate (arrow). Scattered non-specific mucosal thickening is seen in paranasal sinuses (dashed arrows). Coronal post con-

difficulty in sanitization of equipment. Hence, in this clinical scenario, an optimally performed CECT can be acquired for the appropriate demonstration of mucosal disease, bone erosion and destruction, extrasinus extensions, and vascular structures like internal carotid artery (ICA) and cavernous sinuses [7]. In several institutions, non-contrast CT (NCCT) is performed, which can show the extent of the mucosal disease (earliest finding being unilateral nasal cavity soft tissue thickening) and most of the complications, except the vascular complications. Apart from evaluation of suspected intracranial and intraorbital disease extension, MRI can also be used in follow-up imaging, and equivocal CT findings. In addition, non-contrast MRI can be used in those patients with deranged renal function tests. The suggested steps while evaluating a contrast enhanced study for suspected AIFR are as follows.

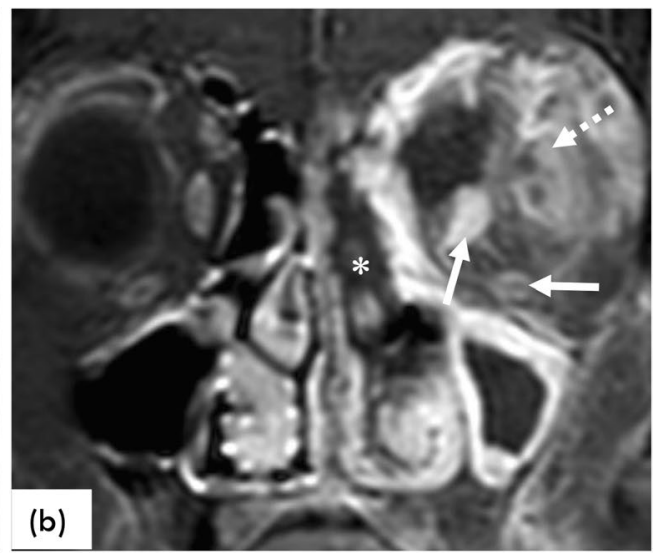

trast T1WI (b) reveals no enhancement of left middle turbinate $\left(^{*}\right)$ : "Black turbinate sign." Extensive left orbital involvement in the form of thickened muscles (arrows) and fat stranding (dashed arrow) is also seen

\section{Step 1 [Establishing diagnosis (AIFR versus chronic sinusitis)]}

The first step is to rule out other etiologies of sinonasal complaints and establish the diagnosis of AIFR. Few patients may have pre-existing chronic rhinosinusitis (CRS) or CRS with polyposis (CRSwP). These would have typical imaging appearance of mucosal thickening, retention cysts, and polyposis (Fig. 1a and b) along with thickening of the bony walls. Chronic rhinosinusitis/chronic fungal colonization would not have extrasinus soft tissue or bony destruction. Similarly, patients may have other forms of chronic fungal disease such as mycetoma or allergic fungal sinusitis [8]. Mycetoma is a mass like, noninvasive lesion composed of fungal hyphae (Aspergillus fumigatus) usually involving a single sinus. An expanded
Fig. 3 CAM causing oroantral fistula. Coronal CT in bone window (a) reveals mucosal disease in bilateral maxillary and left ethmoidal sinuses with erosion of alveolar process of maxilla (*). Sagittal image (b) shows erosion of alveolar process of maxilla (*) and hard palate (arrowhead) with formation of oronasal fistulas (arrows)
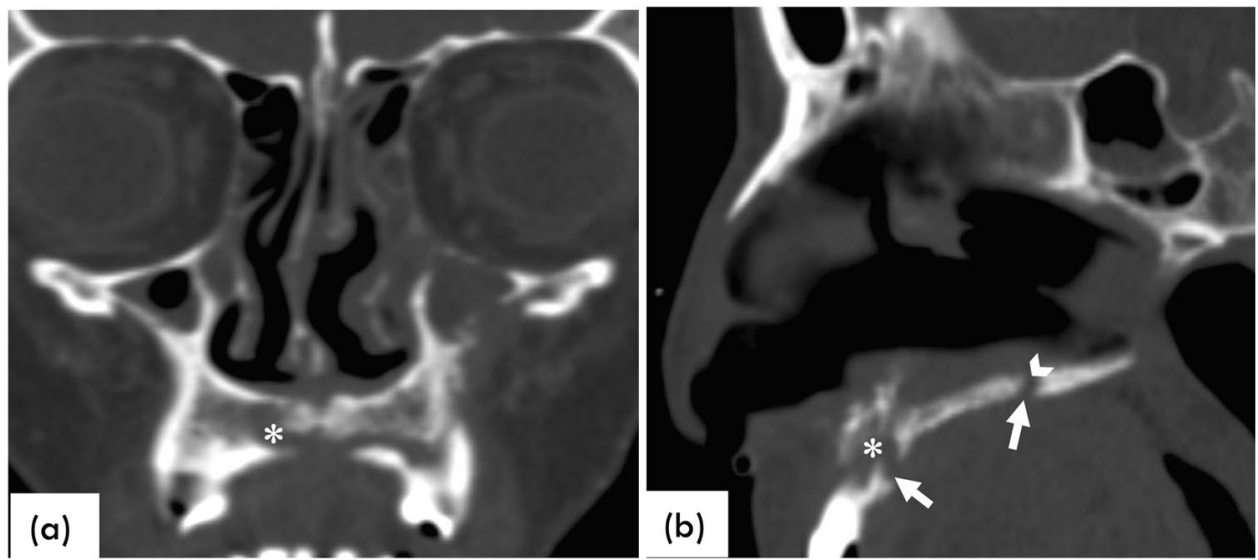

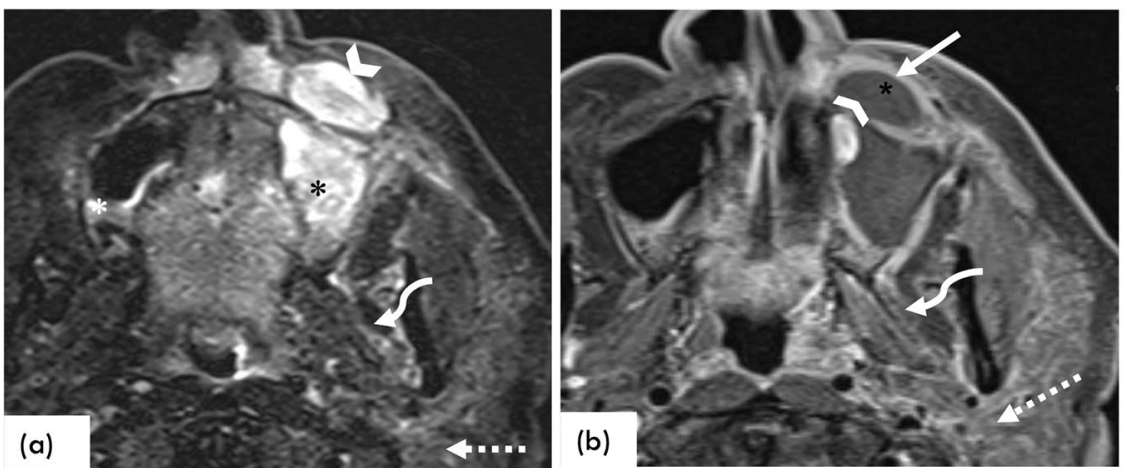

(b)

Fig. 4 CAM with anterior perisinus involvement. Axial T2FS MRI (a) reveals bilateral maxillary sinusitis (*) with an abscess (arrowhead) in left preantral soft tissue. On post contrast T1WI (b), wall of the abscess shows enhancement (straight arrow). Inflammatory changes are seen in left infratemporal fossa (curved arrow) and left parotid space (dashed arrow). Axial CECT image in another patient (c) shows a necrotic abscess in the left preantral soft tissue. Wall of the abscess shows enhancement (arrowhead) and multiple air foci are seen within the abscess (arrow). Mucosal thickening is seen in left maxillary sinus $(*)$ with erosion of anterior wall of left maxillary sinus (curved arrow). Inflammatory soft tissue is seen in left infratemporal fossa (block arrow) sinus with internal soft tissue contents (hypodense on CT with central areas of hyperdensity, possibly containing calcifications and hypointense on T2WI in MRI) with peripheral mucosal enhancement are the imaging characteristics of mycetoma (Fig. 1c and d).

$A I F R$, on the other hand, is characterized by sinonasal mucosal thickening associated with bone erosions and extrasinus spread of the disease. It is important to recognize the early CT finding of unilateral nasal cavity soft tissue in AIFR. This extrasinus spread may also occur with intact bony walls likely through microvascular channels in the bone as seen in involvement of the posterior periantral fat [7]. The perisinus spread is characteristic and may also involve the pterygopalatine fossa
Fig. 5 CAM with posterior perisinus and posterior deep spaces of neck involvement. Axial CECT image in soft tissue (a) and bone window (b) shows mucosal disease in sphenoid, right maxillary, and bilateral ethmoid sinuses, right nasal cavity. Enhancing soft tissue is seen in right pterygopalatine fossa (arrow) with extension into sphenopalatine foramen (dashed arrow) and Vidian canal (curved arrow) without any bony erosion. Axial MRI images show mucosal thickening in right maxillary sinus (*), appearing hyperintense on T2FS image (c) and showing enhancement on contrast T1WI (d). There are inflammatory changes in right postantral region (arrowhead), right pterygopalatine fossa (straight arrow), bilateral infratemporal fossa (dashed arrows), and right carotid space (curved arrow in d)
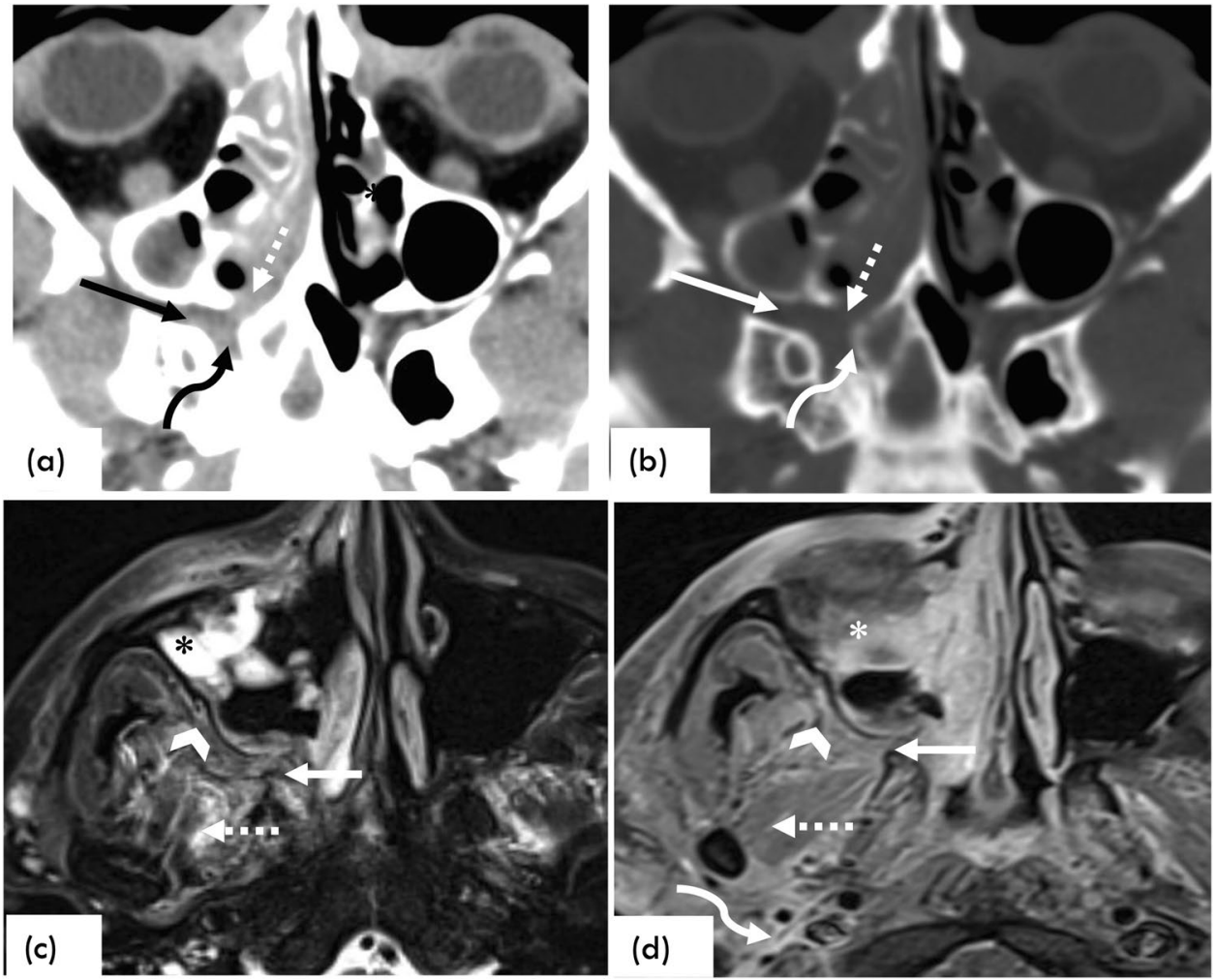
Table 2 Extent of orbital involvement and intracranial disease in ROCM

\begin{tabular}{ll}
\hline Orbital involvement & Intracranial disease \\
\hline - Inflammation of the nasolacrimal duct and sac & $\bullet$ Cavernous sinus invasion \\
- Thickening of the medial rectus (early sign) & $\circ$ Bulky cavernous sinus \\
- Enlargement of all extraocular muscles & $\circ$ Internal hypodensity on CECT \\
- Retrorbital fat stranding & $\circ$ Altered signal intensity on MRI \\
- Bone erosion of lamina papyracea, inferior orbital wall & $\circ$ Dilated superior ophthalmic vein \\
- Optic nerve: stretched, thickened, enhancing walls & $\bullet$ Meningeal or intraxial infection \\
- Soft tissue in orbital apex, superior orbital fissure, inferior orbital fissure & $\circ$ Anterior skull base erosion (cribriform plate) \\
- Uveoscleral thickening (panophthalmitis) & $\circ$ Leptomeningitis \\
- Tenting of the posterior pole of the globe (guitar pick sign) & $\circ$ Cerebritis \\
- Bilateral disease & $\circ$ Intracranial abscess (peripherally enhancing, low density lesions) \\
& $\bullet$ Vascular complications \\
& $\circ$ Arterial narrowing \\
& $\circ$ Arteritis \\
& $\circ$ Aneurysm \\
& $\circ$ Large vessel infarction
\end{tabular}

and infratemporal fossa and is believed to be along the sphenopalatine artery or posterior superior nasal nerves.
Intraorbital and intracranial extensions are the most dreaded complications.
Fig. 6 CAM with orbital involvement (lacrimal apparatus) in two patients. CECT coronal image in soft tissue window (a) shows enhancing soft tissue in the region of left lacrimal sac (*), along with opacification of left frontal sinus (arrow) and nasal cavity (arrowhead). b-d CECT coronal (b) and axial (c and d) images in another patient showing soft tissue in bilateral nasolacrimal duct (arrow) with expansion of left nasolacrimal duct. There is partial opacification of bilateral maxillary $(*)$ and left ethmoid sinuses (arrowhead in (b)). CECT axial image in bone window (d) shows erosion of posterior wall of left nasolacrimal duct (arrow). Bony walls of right nasolacrimal duct (dashed arrow) are normal. There are erosions of posterior wall of left maxillary sinus (curved arrow), left lateral (arrowhead), and medial (block arrow) pterygoid plates
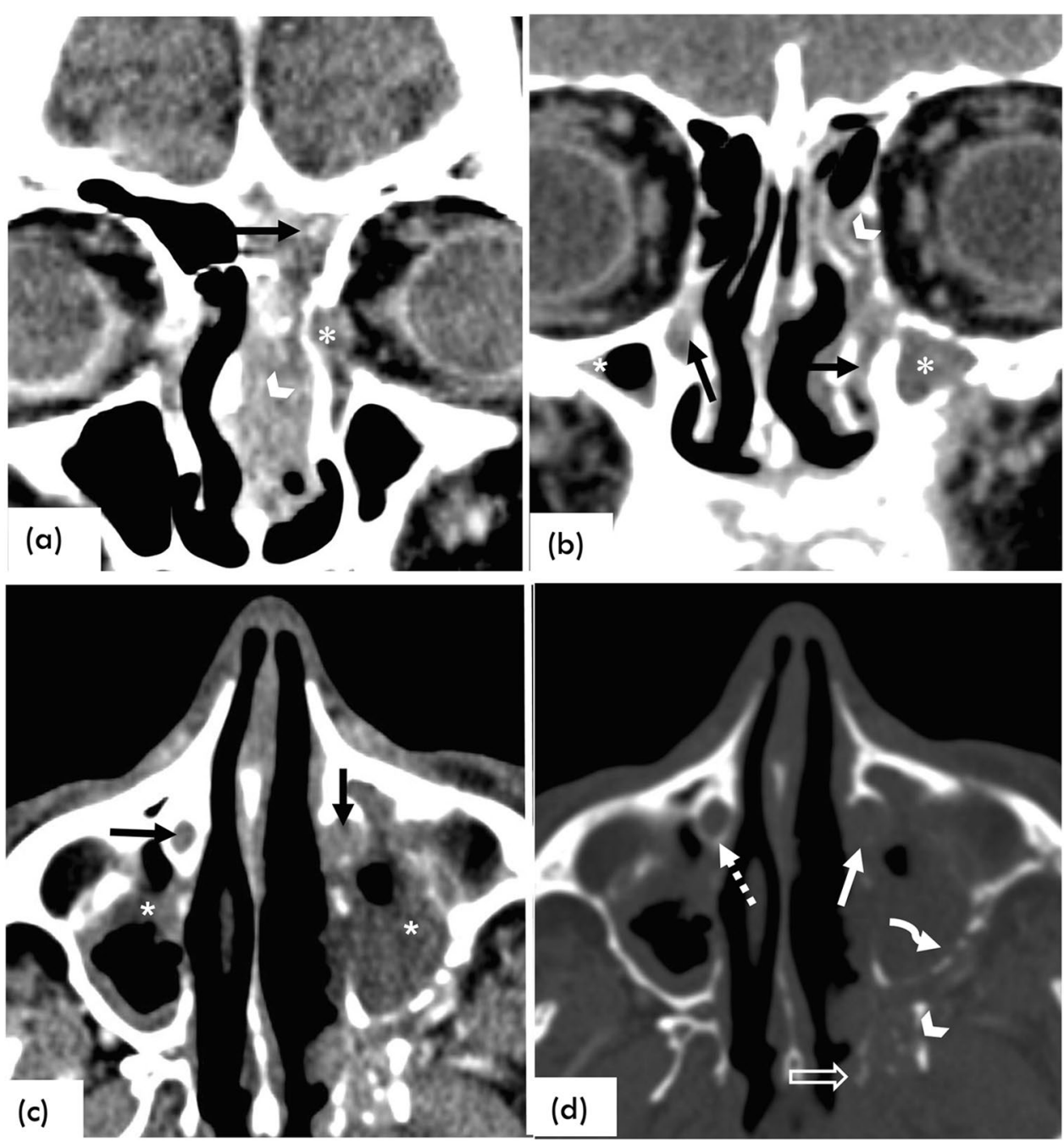

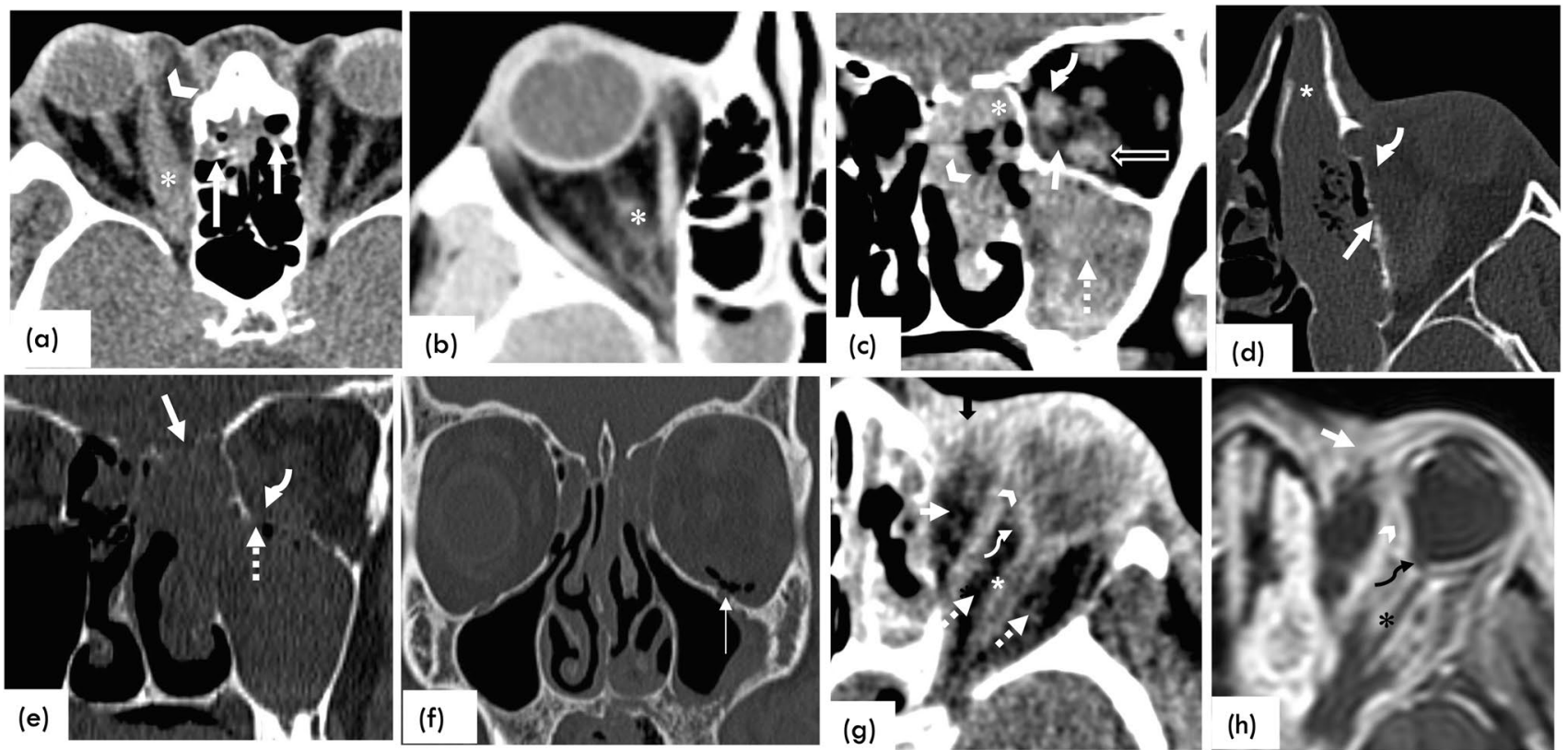

Fig. 7 CAM with a spectrum of orbital involvement (extraconal and intraconal compartments). CECT axial image in soft tissue window (a) shows opacification of bilateral anterior ethmoid air cells (arrow) with associated inflammatory soft tissue (arrowhead) in medial extraconal compartment of right globe and bulky right medial rectus muscle $(*)$.There is proptosis of right globe. CECT axial image in soft tissue window (b) shows fat stranding (*) in the retrobulbar intraconal compartment of right orbit with proptosis of right globe. CECT coronal image (c) shows inflammatory soft tissue (arrow) in inferomedial part of left orbit with bulky left medial rectus (curved arrow) and inferior rectus (block arrow). There is opacification of right maxillary sinus (dashed arrow), ethmoid sinus (*), and nasal cavity (arrowhead). Axial CT (d) showing erosion of left lamina papyracea (arrow). There is opacification of left nasal cavity $(*)$ and

\section{Step 2 [Delineation of disease extent and important complications]}

The next step on imaging is to completely defines the extent of the disease. This has important clinical implications as imaging provides a road-map for the degree of surgical debridement.

The initial stage of disease (Table 1) commonly involves the nasal mucosa with soft tissue thickening and ulceration and bony erosion of the nasal septum. The middle turbinate (Fig. 2a) is usually involved first followed by the involvement of the inferior turbinate or ostium of the nasolacrimal duct [9]. On CECT, this would be seen as mucosal thickening, erosion of bony turbinates with lack of mucosal enhancement; or as the "black turbinate sign" on CEMRI (Fig. 2b).

This is followed by involvement of paranasal sinuses, seen as partial or complete opacification of the sinuses. The disease may spread to the adjacent palate or oral cavity with bone erosions and formation of oronasal or oroantral fistulae (Fig. 3). The further spread of soft tissue inflammation ethmoid sinus with inflammatory soft tissue in medial part of the orbit (curved arrow). Coronal image in bone window (e) shows erosion of cribriform plate (arrow) and inferior wall of left orbit (dashed arrow) and extraconal inflammatory soft tissue (curved arrow). Coronal image in bone window (f) shows air foci in inferior part of the orbit suggestive of orbital emphysema (arrow). Axial CECT (g) and post contrast T1WI (h) reveals preseptal, periorbital inflammatory soft tissue (block arrow) around left globe, along with thickening and enhancement of coats of the globe on the medial aspect (arrowhead). Fat stranding is seen in medial extraconal compartment (arrow in $\mathbf{g}$ ) and retrobulbar intraconal compartment (dashed arrow in g). There is tenting of globe posteriorly (curved arrow): "Guitar-pick sign." Right optic nerve is stretched with enhancement of optic nerve sheath of intraorbital part of optic nerve $\left(^{*}\right)$

can be to the anterior (Fig. 4) and posterior periantral space, seen as obliteration of the fat with inflammatory edema. This may be followed by necrotic abscess formation in the buccal space and bony erosions or marrow edema of the zygoma and mandible. Spread of disease (Table 1) from the posterior nasal cavity can be to the nasopharynx, sphenopalatine foramen, pterygopalatine fossa, and infratemporal fossa (Fig. 5) [7, 10].

Early involvement of the orbit (Table 2) is characterized by inflammation of the nasolacrimal duct and sac (Fig. 6). Thickening of the medial rectus is also an early sign and maybe followed by enlargement of rest of the extraocular muscles $[9,10]$. Retrorbital fat stranding or patchy enhancement can be seen especially along the medial and inferior orbital fat. Bone erosion of the lamina papyracea and all orbital walls should be carefully looked for. The optic nerve can be stretched and thickened due to inflammatory edema (Fig. 7). The soft tissue inflammation can spread to the orbital apex, superior orbital fissure, and inferior orbital fissure (Fig. 8). Uveoscleral thickening or tenting of the posterior pole of the globe (guitar-pick sign) indicates severe 
inflammatory edema and/or orbital compartment syndrome (Fig. 7) [11].

Spread of disease from the orbital apex further leads to cavernous sinus invasion (Table 2). On imaging (Fig. 9), this can be seen as bulky cavernous sinus, internal hypoenhancement (suggestive of thrombosis), or altered signal intensity of cavernous sinus on MRI. Dilated superior ophthalmic vein is an indirect sign of cavernous sinus thrombosis $[10,11]$. Intracranial complications (Fig. 10) include leptomeningitis or cerebritis and intracranial abscess (peripherally enhancing, low density lesions) formation. This is frequently seen along the basifrontal lobe (anterior skull base or cribriform plate erosion) and temporal lobes. Also, because of the angioinvasive property of the fungus, there is a tendency of arterial narrowing, arteritis, with aneurysm formation (Fig. 9). The intracranial part of ICA, especially the cavernous portion, should be carefully evaluated for any narrowing. There may be large vessel infarction seen as areas of altered signal intensity with diffusion restriction on MRI [10]. MR angiography can document the degree of arterial involvement, which can be well depicted on the volume rendered images.

\section{Implications of defining extent of disease in patient management}

AIFR is a potentially life-threatening condition and hence, timely diagnosis and treatment is essential. This includes aggressive medical management (amphotericin B in mucormycosis and voriconazole in aspergillosis), appropriate surgical debridement, and control of the comorbid conditions. Disease limited to the nasal cavity or sinuses is treated with endoscopic or combined approach. This includes turbinectomy, wide middle meatal antrostomy, ethmoidectomy, and sphenoidotomy [9]. Palatal necrosis is managed by infrastructure maxillectomy or palatectomy according to the extent of necrosis. Orbital extension is managed by varied surgical approaches including endoscopic subperiosteal abscess evacuation, orbital decompression, orbitotomy, and orbital exenteration [4]. This is usually accompanied by aggressive sinus debridement and sinus irrigation with amphotericin B [9]. Intracranial abscess may require neurosurgical intervention if systemic condition permits. Few cases develop progressive symptoms post locoregional debridement and need follow-up MRI to document residual and progressive lesions (Fig. 11).

\section{Step 3 [Mucormycosis versus aspergillosis]}

The diagnosis of AIFR is primarily based on nasal endoscopy and biopsy which also gives the etiological organism. Though it is difficult to definitely suggest aspergillosis or mucormycosis on imaging, there are few signs which help raise a diagnostic possibility. Both Aspergillus and Mucor commonly affect the ethmoid sinuses and are characterized by mucosal thickening. Complete opacification or air-fluid levels may also be seen
Fig. 8 CAM with orbital apex involvement. Axial CECT in soft tissue window (a) reveals inflammatory soft tissue $(*)$ in the region of right orbital apex. Proptosis of right globe is also seen (arrow). Coronal CECT (b) shows soft tissue in the region of left orbital apex (*). Axial CECT (c) shows inflammatory soft tissue in the region of right inferior orbital fissure (arrow). Left inferior orbital fissure is normal (arrowhead). There is opacification of sphenoid sinus $(*)$ and left ethmoid sinus. CECT coronal image (d) shows extension of inflammatory soft tissue in right inferior orbital fissure (arrow) and right infratemporal fossa (dashed arrow) with opacification of sphenoid sinus $(*)$
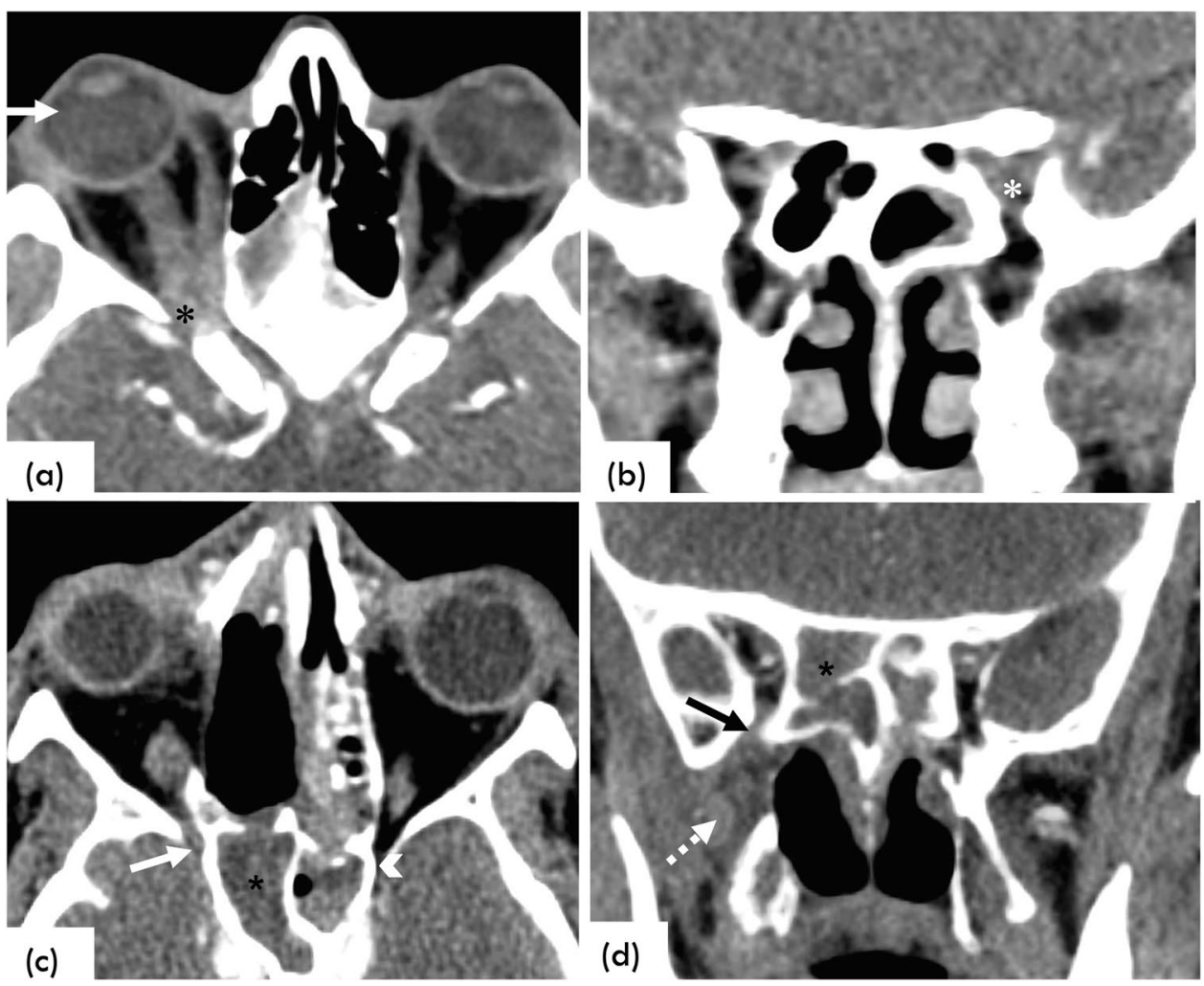


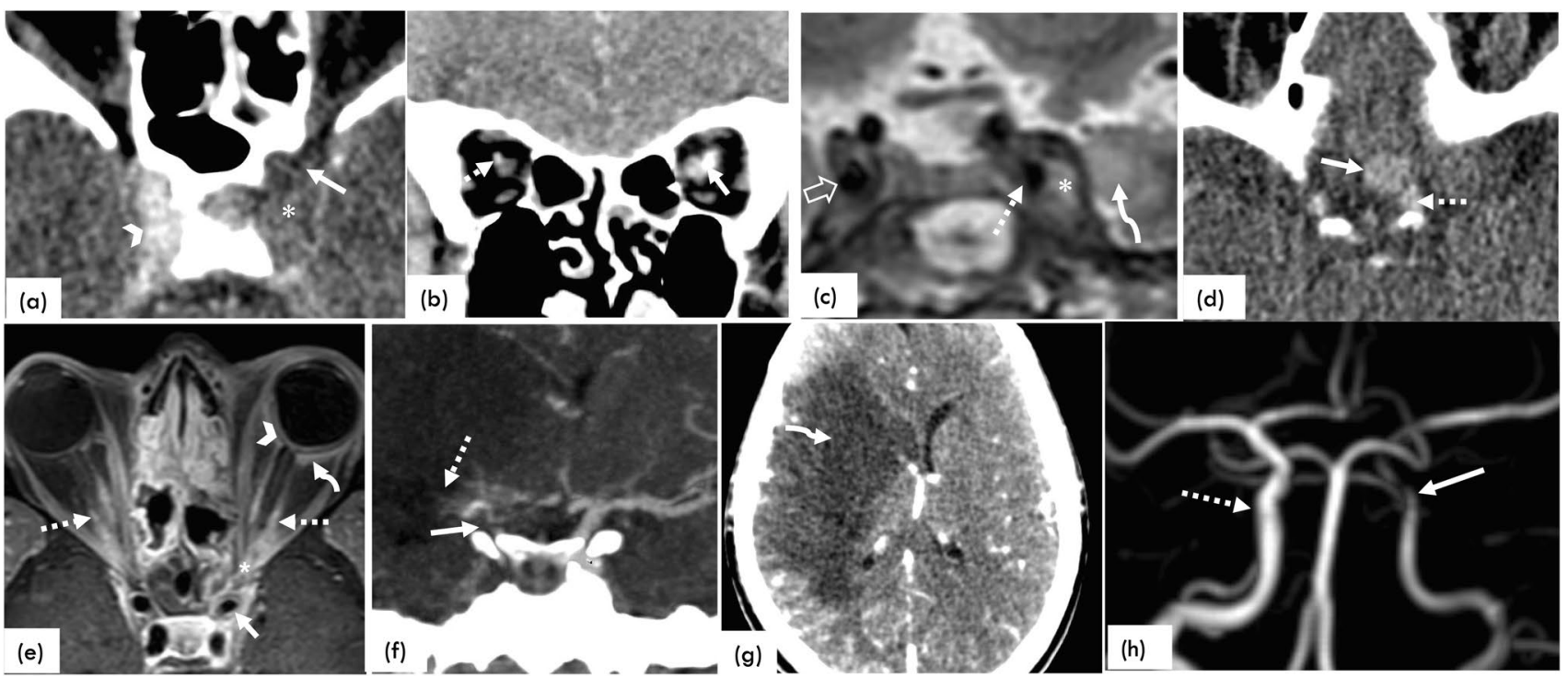

Fig. 9 CAM with intracranial vascular complications. Axial CECT (a) shows bulky left cavernous sinus showing no enhancement $\left(^{*}\right)$, right cavernous sinus is normal (arrowhead). There is hypodense soft tissue anterior to the left cavernous sinus in the region of left orbital apex (arrow). Coronal image (b) shows prominent left superior ophthalmic vein (arrow). Right superior ophthalmic vein is normal (dashed arrow). Coronal T2WI (c) shows bulky left cavernous sinus with hyperintense signal in the lateral part of the sinus (*). There is narrowing of cavernous segment of left internal carotid artery (dashed arrow). Focal area of hyperintensity is seen in left temporal lobe (curved arrow) in (c) suggestive of edema. Right internal carotid artery is normal (block arrow). Axial CECT (d) shows a saccular aneurysm (arrow) in suprasellar cistern arising from anteromedial wall of supraclinoid segment of left internal carotid artery (dashed arrow). Axial gadolinium-enhanced T1WI (e) shows bulky left cav- ernous sinus with enhancement of wall of cavernous segment of left internal carotid artery suggestive of periarteritis (arrow). There is narrowing of cavernous segment of left internal carotid artery. Enhancing soft tissue is seen in the region of left orbital apex $(*)$. Bilateral optic nerve sheath enhancement is also seen (dashed arrow) with enhancement in the retrobulbar region of left orbit (curved arrow) and early posterior tenting of left globe (arrowhead). Coronal CECT image in MIP projection (f) shows thrombosis of right internal carotid artery (arrow) and right middle cerebral artery (dashed arrow) and the axial CECT brain image shows a large area of hypodensity in right middle cerebral artery territory suggestive of acute infarct (curved arrow). 3D CEMRA image in coronal (h) plane shows narrowing of cavernous segment of left internal carotid artery (arrow). Right ICA shows normal calibre and contrast enhancement (dashed arrow)
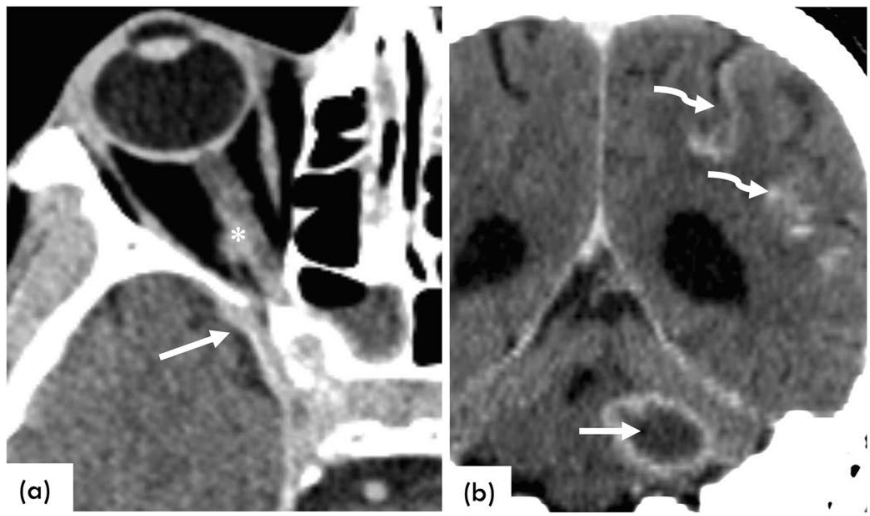

Fig. 10 CAM with intracranial complications. Axial CECT (a) reveals dural thickening and enhancement along right temporal lobe (arrow). There is enhancement of intraorbital part of right optic nerve (*). Coronal CECT (b) reveals intracranial abscess (arrow) in left cerebellar hemisphere and left frontoparietal leptomeningeal enhancement (curved arrows). Coronal T2WI (c) of a patient post right orbital
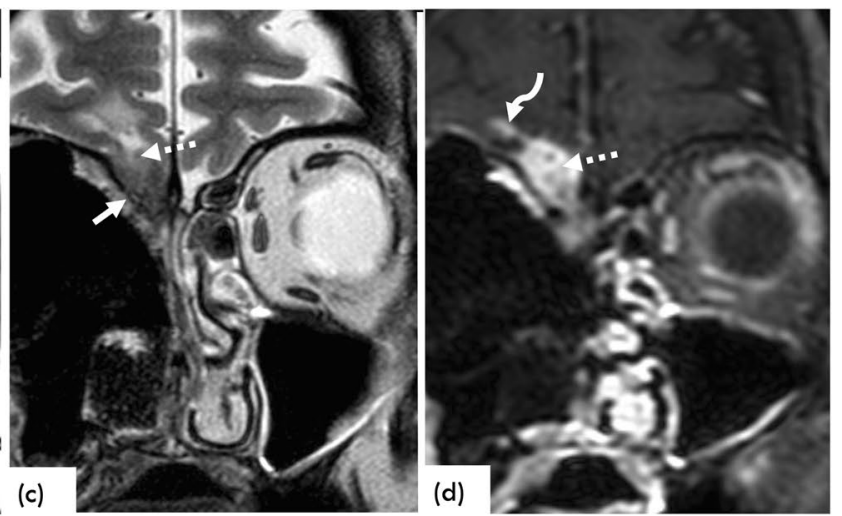

exenteration shows erosion of right cribriform plate (arrow) with heterogenous hypointense extraaxial soft tissue (dashed arrow) along the right basifrontal region, showing enhancement on gadoliniumenhanced T1WI (d). Focal leptomeningeal enhancement (curved arrow) is also seen along the right frontal lobe 
Fig. 11 Postoperative appearance in case of rhino-orbital CAM. CECT sagittal (a) and coronal (b) images of the patient post left orbital exenteraabscess (arrow in $\mathbf{a}$ and $\mathbf{b}$ ) in left temporal lobe along with dural enhancement (block arrow in a) along left temporal lobe suggestive of intracranial extension of the disease tion $\left(^{*}\right)$ show an intracranial

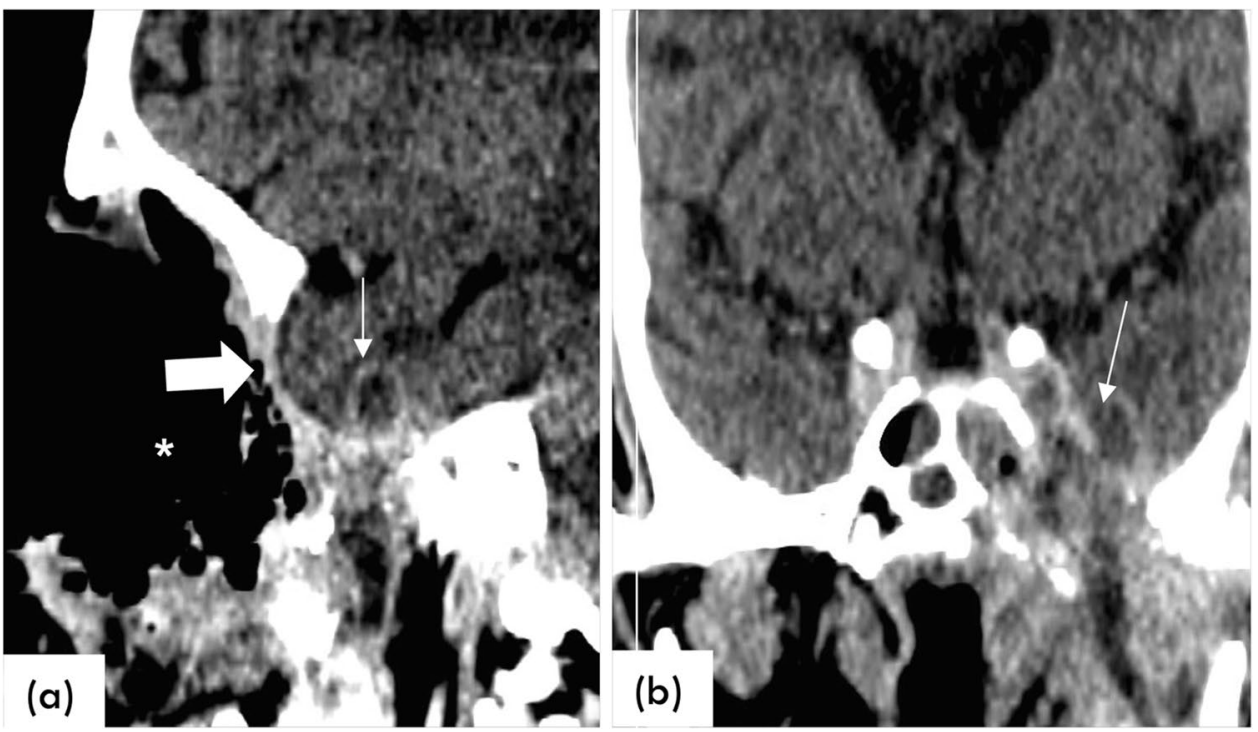

(a) Ir

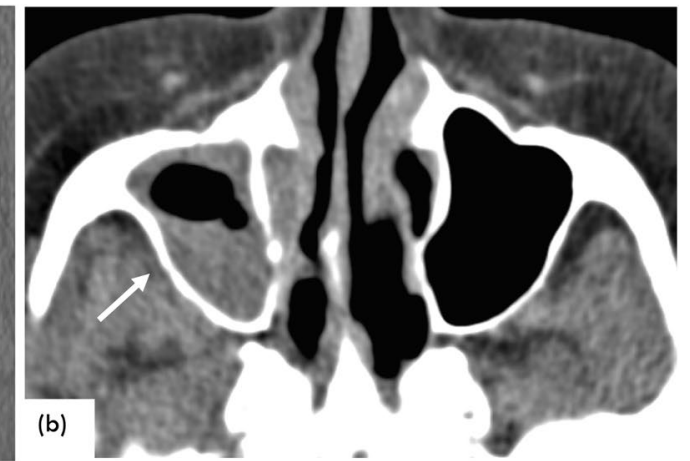

(a)

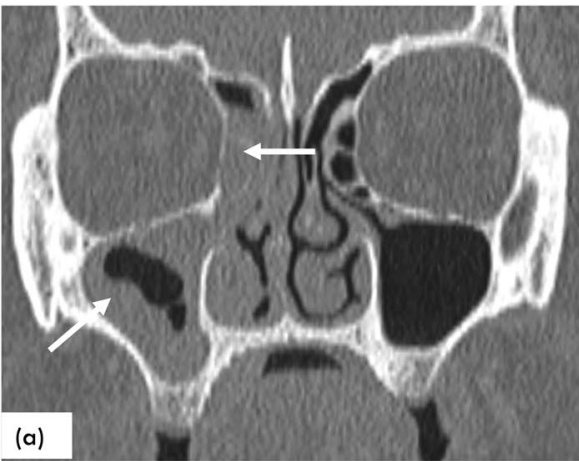

Fig. 12 Acute invasive fungal rhinosinusitis (unilateral disease) due to aspergillosis in a COVID-19 patient. NCCT coronal image in bone window (a) and axial image in soft tissue window (b) show mucosal

in AIFR. Aspergillus is reported to have sino-cranial disease in the absence of orbital involvement [12], with dural-based masses which are homogenous, hypointense on T2WI, and show moderate post contrast enhancement. Aspergillus has a tendency towards unilateral involvement (Fig. 12) compared to Mucor [13]. Mucor, however, has a marked tendency to cause invasive thrombosis of vessel walls and the characteristic "black turbinate sign" (non-enhancement of nasal mucosa or turbinates on CECT/CEMRI) [14]. It is also characterized by irregular enhancement with necrotic soft tissue lesions (Fig. 4).

\section{Conclusion}

Sinonasal AIFR is a potentially life-threatening, invasive fungal infection. Imaging plays a key role in the early diagnosis and mapping of disease extent, which are essential for appropriate treatment. Timely initiation of antifungal thickening within right maxillary sinus and ethmoid sinus (arrows in a) with loss of posterior periantral fat pad (arrow in b). There is rarefaction of ethmoid trabeculae but no orbital involvement

therapy and surgical intervention can significantly improve clinical outcomes.

Data availability NA

Code availability NA

\section{Declarations}

Conflict of interest The authors declare that they have no conflict of interest.

1. Pal R, Singh B, Bhadada SK, Banerjee M, Bhogal RS, Hage N, Kumar A (2021) COVID-19-associated mucormycosis: an

\section{References}


updated systematic review of literature. Mycoses. https://doi.org/ 10.1111/myc. 13338

2. Sharma S, Grover M, Bhargava S, Samdani S, Kataria T (2021) Post coronavirus disease mucormycosis: a deadly addition to the pandemic spectrum. J Laryngol Otol 135(5):442-447. https://doi. org/10.1017/S0022215121000992

3. Orlowski HLP, McWilliams S, Mellnick VM, Bhalla S, Lubner MG, Pickhardt PJ, Menias CO (2017) Imaging spectrum of invasive fungal and fungal-like infections. Radiographics 37(4):11191134. https://doi.org/10.1148/rg.2017160110

4. El-Kholy NA, El-Fattah AMA, Khafagy YW (2021) Invasive fungal sinusitis in post COVID-19 patients: a new clinical entity. Laryngoscope. https://doi.org/10.1002/lary.29632

5. Skied A, Pavleas I, Drogari-Apiranthitou M (2020) Epidemiology and diagnosis of mucormycosis: an update. J Fungi (Basel) 6:265. https://doi.org/10.3390/jof6040265

6. Cornely OA, Alastruey-Izquierdo A, Arenz D, Chen SCA, Dannaoui E, Hochhegger B et al (2019) Global guideline for the diagnosis and management of mucormycosis: an initiative of the European Confederation of Medical Mycology in cooperation with the Mycoses Study Group Education and Research Consortium. Lancet Infect Dis 19:e405-e421. https://doi.org/10.1016/S14733099(19)30312-3

7. Middlebrooks EH, Frost CJ, De Jesus RO, Massini TC, Schmalfuss IM, Mancuso AA (2015) Acute invasive fungal rhinosinusitis: a comprehensive update of CT findings and design of an effective diagnostic imaging model. AJNR Am J Neuroradiol 36(8):1529-1535. https://doi.org/10.3174/ajnr.A4298

8. Deutsch PG, Whittaker J, Prasad S (2019) Invasive and non-invasive fungal rhinosinusitis - a review and update of the evidence. Medicina (Kaunas) 55(7):319. https://doi.org/10.3390/medicina55 070319
9. Honavar SG (2021) Code Mucor: guidelines for the diagnosis, staging and management of rhino-orbito-cerebral mucormycosis in the setting of COVID-19. Indian J Ophthalmol 69(6):13611365. https://doi.org/10.4103/ijo.IJO_1165_21

10. Gorovoy IR, Kazanjian M, Kersten RC, Kim HJ, Vagefi MR (2012) Fungal rhinosinusitis and imaging modalities. Saudi J Ophthalmol 26(4):419-426. https://doi.org/10.1016/j.sjopt.2012.08. 009

11. Nguyen VD, Singh AK, Altmeyer WB, Tantiwongkosi B (2017) Demystifying orbital emergencies: a pictorial review. Radiographics 37:947-962. https://doi.org/10.1148/rg.2017160119

12. Bhalla D, Bhalla AS, Manchanda S (2021) Can imaging suggest the aetiology in skull base osteomyelitis? A systematic literature review. Pol J Radiol 86:e309-e321. https://doi.org/10.5114/pjr. 2021.106470

13. Slonimsky G, McGinn JD, Goyal N, Crist H, Hennessy M, Gagnon E, Slonimsky E (2020) A model for classification of invasive fungal rhinosinusitis by computed tomography. Sci Rep 10(1):12591. https://doi.org/10.1038/s41598-020-69446-5

14. Ashour MM, Abdelaziz TT, Ashour DM, Askoura A, Saleh MI, Mahmoud MS (2021) Imaging spectrum of acute invasive fungal rhino-orbital-cerebral sinusitis in COVID-19 patients: a case series and a review of literature. J Neuroradiol S01509861(21):00130-00139. https://doi.org/10.1016/j.neurad.2021. 05.007

Publisher's note Springer Nature remains neutral with regard to jurisdictional claims in published maps and institutional affiliations. 